\title{
On the applicability of viscosity-based capillary bundle concepts to predict the penetration behaviour of polymer solutions into sand
}

\author{
Rowena Verst $^{1}$ (D) $\cdot$ Wolfgang Lieske $^{2} \cdot$ Wiebke Baille $^{2} \cdot$ Matthias Pulsfort $^{1} \cdot$ Torsten Wichtmann $^{2}$
}

Received: 30 January 2020 / Accepted: 3 May 2021 / Published online: 10 June 2021

(C) The Author(s) 2021

\begin{abstract}
The increasing use of polymer solutions as support fluids in pile drilling, diaphragm walling or tunnelling applications demands a more detailed discussion of their penetration behaviour and prediction thereof. In this context, the capillary bundle approach can be a useful tool. However, while it is widely discussed in the oil and gas application, the subject is currently addressed only scarcely with regard to support fluid penetration targeting stagnation, where small flow velocities and non-cohesive soil environments are of relevance. In these boundary conditions, the applicability of capillary bundle approaches is not yet sufficiently confirmed and substantiated. The current paper thus reviews current capillary bundle models based on Hagen-Poiseuille in combination with a power-law rheological model and discusses their applicability with respect to support fluid application in the context of experimental soil permeation tests for small gradients $(i \leq 10)$. Two granular materials of similar grain size, but different angularity (glass beads and sand), and four polymer solutions varying in polymer chain length and concentration are investigated, and the impact of model assumptions and bulk material input variables is systematically discussed. The experimental results show that the theoretical models are generally able to predict the filter velocity qualitatively for values above $\bar{v}=5 \times 10^{-7} \mathrm{~m} / \mathrm{s}$ and also quantitatively, when an empirical shift factor $\alpha^{*}$ is introduced and water permeability values are determined experimentally. With respect to the influence of soil parameters, it was found that the soil particle roughness decreases the flow velocity of the polymer solution despite similar hydraulic conductivity in water. Polymer chain length and concentration were observed to control the degree of possible dilution $\left(\alpha^{*}<1\right)$ in the porous system compared to bulk rheological characteristics. It can therefore be concluded that capillary bundle models can indeed be applied in the targeted fields even though they are unable to predict a complete stagnation for $i>0$. However, rather than specific model assumptions for tortuosity, taking into account the specific soilpolymer interaction has shown to be of primary importance to ensure no under- or over-prediction of penetration velocities solely based on bulk rheology.
\end{abstract}

Keywords Bulk rheology · Fluid penetration into granular material · In-situ rheology $\cdot$ Polymer solutions · Support fluids

Rowena Verst

verst@uni-wuppertal.de

Wolfgang Lieske

wolfgang.lieske@rub.de

Wiebke Baille

wiebke.baille@rub.de

Matthias Pulsfort

pulsfort@uni-wuppertal.de

Torsten Wichtmann

torsten.wichtmann@rub.de

Bergische Universität Wuppertal, Wuppertal, Germany

2 Ruhr-Universität Bochum, Bochum, Germany

\section{Introduction}

The viscosity of a fluid has a crucial influence on the respective permeability of soils. In several geotechnical and geoenvironmental applications, fluids with viscosity values much greater than that of water are injected into the ground for different purposes.

Particularly the use of water-soluble polymers (i.e. linear, unbranched macromolecules of high molar mass) as a viscosity-modifier has gained considerable attention for polymer flooding in enhanced oil recovery (EOR) and for the fluid-supported construction of drilled piles, diaphragm 
walls or tunnels. This has particularly been the case since the use of polymer solutions is associated with several advantages compared to oil- or bentonite-based supporting agents, e.g. concerning environmental sustainability, chemical stability and better regeneration properties [5, 21, 28]. Viscous polymeric pore fluids have also been used in physical modelling of centrifuges to account for scale effects [30].

However, a quantitative prediction of the penetration behaviour of polymer solutions into the porous structure of soil (in-situ flow behaviour) poses several challenges due to the complex interaction between water, polymer and soil. Current modelling approaches therefore generally idealise the interaction as a system of two phases: fluid, defined by its bulk rheology, and porous medium, represented by a set of capillary tubes or networks. This simplification is generally found to be sufficiently accurate for engineering practice with water $[8,15,20]$ as well as water and polymer $[3,28]$. These approaches are especially fairly established for numerical modelling of polymer flooding [19, 24, 28, 33]

First concepts exist to apply capillary bundle models for the prediction of the penetration behaviour of polymer support fluids for the stabilisation of earth walls [22, 29]. Yet, it is known, that the polymer chains' exposure to shear and therefore viscosity mobilisation and thus penetration behaviour differs in a porous system with varying size of flow paths, tortuosity and surface roughness [1,27], and the geotechnical boundary conditions may differ distinctly from EOR applications [29]. Polymer support fluids are designed to, e.g., penetrate as slowly as possible into the natural ground or preferably stagnate. However, the applicability of the common approaches for this purpose is not yet sufficiently confirmed.

The current paper thus presents an experimental study to address the capability of the capillary bundle approach to predict flow velocities with focus on geotechnical applications. Permeability tests are carried out with an exemplary soil grain fraction with varying surface roughness and a distinct range of polymer properties of a typical synthetic polymer to assess possible soil-fluid interaction mechanisms, especially at low flow velocities. Viscosity is studied in a rotational rheometer. In particular, following EOR modelling adaptions, the applicability of empirical shift factors to cover deviations between in-situ and bulk rheology will be studied as part of the model approach with the aim to identify possible systematic relationships.

\section{Background}

The ability of a fluid to conduct through a saturated layer of soil due to a hydraulic gradient is governed by both soil matrix and fluid properties $[3,10,27]$. The former may be separated into effects arising from the arrangement of the grains (e.g. total pore volume, pore size distribution, shape and connectivity of the flow paths) and the surface characteristics of the solids (e.g. roughness, surface charge). The solution with dissolved polymers is usually represented by a homogeneous fluid with a defined bulk rheology and its related polymer characteristics.

Water-soluble polymers are macromolecules consisting of a large number of linearly arranged repeating units. They derive their viscosifying qualities in solution from their ability to stretch or entangle when sheared. Their mechanical shear resistance thereby depends upon entropic and drag forces governed, e.g. by molecule composition, molar mass (chain length), ionicity and concentration in solution $[6,21,27]$.

Most of the fluids found in geotechnical applications such as water or salt solutions can be considered as relatively incompressible, ideal Newtonian fluids with a constant viscosity value under constant temperature and pressure conditions as the contained molecules are relatively small (Fig. 1). Consequently, common soil-mechanical approaches for fluid permeation through soil derived in the last century, so-called engineering or generalized Newtonian approaches, mainly focus on a constant viscosity $\eta$ as the key parameter to describe the shearing flow behaviour in a distinct geometric environment: Darcy's law [15], the Blake-Kozeny equation $[4,20]$ or the Kozeny-Carman equation [8], to name a few.

In contrast, polymer solutions, when tested at stepwise steady-state conditions (laminar flow) by means of rotational rheometers (bulk rheology), exhibit a shear-thinning behaviour. That is, the shear resistance (viscosity) increases significantly with decreasing shear rate $\dot{\gamma}$ (and corresponding flow velocity), when the flexible polymer chains get more entangled. For medium shear rates, the bulk rheology of polymer solutions can be represented adequately by the power-law model $\left(\eta=\tau / \dot{\gamma}=\kappa \cdot \dot{\gamma}^{m-1}\right)$ developed by Ostwald and de Waele [25], which describes an exponential increase of the viscosity $\eta$ with decreasing shear rate $\dot{\gamma}$ (Fig. 1b). Some bulk rheological models add an upper [29] or upper and lower viscosity plateau [9] at very low and very high shear rates, respectively, to take into account the fully coiled or fully stretched conformation of the polymer chain. Shear thickening behaviour, meaning an increase in viscosity with increasing shear rates, has been observed, e.g. by [27] in soil permeation tests (in-situ rheology) for shear rates above $\dot{\gamma}=10 \mathrm{1} / \mathrm{s}$, but the corresponding flow velocities are not relevant for the geotechnical applications considered herein and will therefore not be addressed further.

Analytical engineering models for polymer flow through porous media were presented, e.g. by [7, 13, 18, 27-29]. 


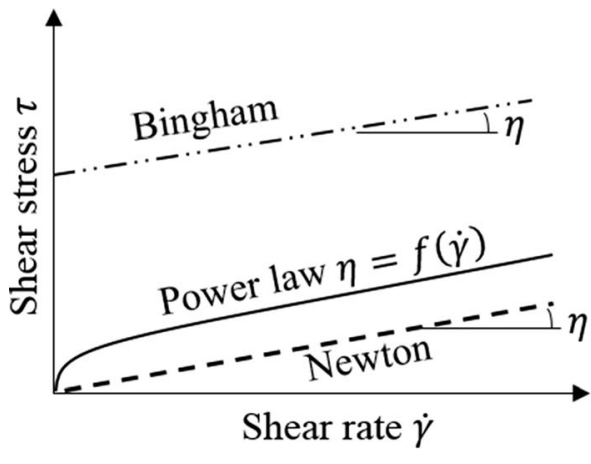

(a) Shear stress

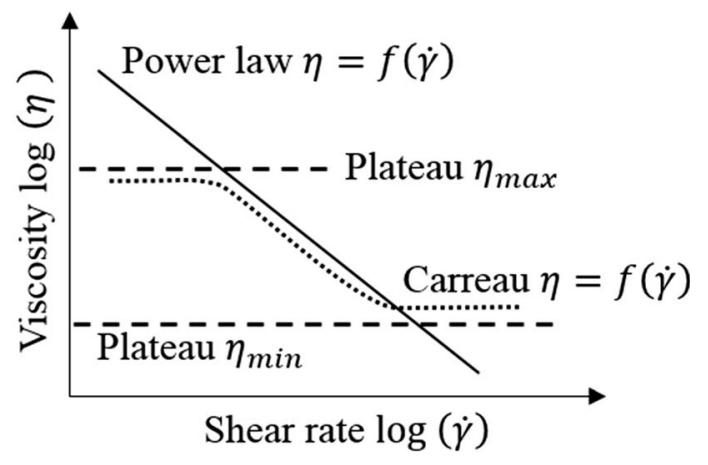

(b) Viscosity

Fig. 1 Typical bulk rheological models for polymer solutions

The main parameters used to describe the flow problem are given in Table 1.

In all cases studied, the predominantly one-dimensional flow through a soil matrix is modelled by a bundle of uniform cylindrical capillaries equal in size by assuming bulk rheological behaviour of the polymer fluid within the pore structure. Laminar flow of a Newtonian fluid with constant viscosity $\eta$ through a single capillary tube according to Hagen-Poiseuille (Eq. 1) with an average capillary flow velocity $\bar{v}_{\text {capillary }}$ is thereby adapted to powerlaw behaviour formulated by Ostwald and de Waele [25] ('OdW') as given by Eq. 2.

$\bar{v}_{\text {capillary }, H P}=\frac{R^{2} \cdot \Delta P}{8 \cdot L \cdot \eta}$

$\bar{v}_{\text {capillary }, \text { OdW }}=\left(\frac{m}{3 \cdot m+1}\right) \cdot R^{\frac{m+1}{m}} \cdot\left(\frac{\Delta P}{2 \cdot L \cdot \kappa}\right)^{\frac{1}{m}}$

The Hagen-Poiseuille term (Eq. 1) can be derived from Eq. 2 with $m=1$ and $\kappa=\eta$. The capillary bundle is equivalent to the soil structure in total pore volume and average flow velocity by defining an equivalent hydraulic radius $R$ for one-dimensional flow (Eq. 3).

$$
R=\sqrt{\frac{8 \cdot K \cdot T}{n}}
$$

The Darcy filter velocity $\bar{v}$ is then calculated from the average pore velocity $\bar{v}_{\text {capillary }}$ according to Dupuit [8] (Eq. 4).

$$
\bar{v}=n \cdot \bar{v}_{\text {capillary }}
$$

The different analytical capillary bundle concepts vary in their assumptions for intrinsic permeability $K$, tortuosity $T$ as well as in the applied rheological model. The considered variations for $\bar{v}$ with reference to a 'basic' model are given in Table 2.

The basic concept uses experimental values for $K$ obtained from flow tests with a reference fluid according to Darcy. This assumption should be valid as long as the reference fluid may be accurately described as an ideal homogeneous fluid with viscosity $\eta$ and specific weight $\gamma$ in all relevant environments, from which the hydraulic conductivity $k$ may be derived (water: $k_{w}=K \cdot \gamma_{w} / \eta_{w}$ ).

The approach by Steinhoff [29] ('ST exp') additionally includes a critical value $\dot{\gamma}_{\text {crit }}$ in order to account for an upper Newtonian plateau, where viscosity is constant

Table 1 Parameter sets for capillary bundle models

\begin{tabular}{lll}
\hline Soil & $D$ & Average grain diameter [m] \\
& $n$ & Porosity [-] (related to void ratio e by: $\left.n=\frac{e}{1+e}\right)$ \\
& $K$ & Intrinsic permeability $\left[\mathrm{m}^{2}\right]$ or \\
& $k_{w}$ & permeability coefficient (water) $[\mathrm{m} / \mathrm{s}]\left(\right.$ related to $K$ by $\left.k_{w}=\frac{K \cdot \gamma_{w}{ }^{a}}{\eta_{w}}\right)$ \\
Polymer solution & $T$ & Tortuosity [-] \\
Test conditions & $\kappa$ & Power-law parameter $[\mathrm{Pa} \mathrm{s}]$ \\
& $m$ & Power-law parameter [-] \\
Fluid-soil interaction & $\Delta H, \Delta P$ & Pressure difference [m, Pa] \\
\hline
\end{tabular}

${ }^{\mathrm{a}}$ Viscosity $\eta_{w}$ and specific density $\gamma_{w}$ from flow tests with water 
$\left(\eta_{\max }=\kappa \cdot \dot{\gamma}_{\text {crit }}^{m-1}\right)$ and the original Hagen-Poiseuille term is used to calculate the respective velocity. This model variation will be used as an example for more complex bulk rheology considerations.

Christopher and Middleman [13] ('CM') based their fundamental formula on the Blake-Kozeny ('BK') approximation for permeability $K$, which assumes ideal spherical particles and thus derives the permeability from the mean grain diameter $D$ and the porosity $n$ of the permeated soil. Moreover, a tortuosity value of $T=\frac{25}{12}$ is applied in accordance with BK. The BK intrinsic permeability term is equal to the respective Kozeny-Carman formula with the exception that [8] additionally applied a tortuosity value of $T=\sqrt{2}$ to the Dupuit calculation (Eq. 4) and thus ended with a slightly different denominator for $K$ compared to BK (180 instead of 150). Christopher and Middleman [13] also allow the possibility to derive $K$ experimentally ('exp') as in the basic model. Unlike other approaches, however, the CM model originally considers $T$ not only within the equivalent hydraulic radius $R$ of the capillary bundle model (Eq. 3), but also within the permeated length $L^{*}=L \cdot T$ (Eq. 2). An additional concept variation ' $\mathrm{CM}$ exp*' will therefore be taken into account, which applies $T$ only to the permeability term and not to the term for the permeated length (as ' $\mathrm{CM} B \mathrm{BK}$ ' and 'CM exp' do) to allow for a differentiation of this effect.

Moreover, there is general agreement that an additional shape or shift parameter is needed to account for deviations between capillary and porous medium apparent shear rate, often visualised as a constant correction ('shift') factor within the double-logarithmic rheology plot $(\dot{\gamma}$ vs. $\eta$ ) [7, 12, 18, 27, 28]. Sorbie [28] summarised findings for xanthan gum solutions which describe the correction factor to be an experimental value differing with varying porous structures. Chauveteau et al. [12], among others, explained these deviations by wall effects creating local depletion

Table 2 Considered capillary bundle concept variations for $\bar{v}$ based on Eqs. 2-4

\begin{tabular}{llll}
\hline & $K\left[\mathrm{~m}^{2}\right]$ & $T[-]$ & Rheology \\
\hline 'Basic' & $\frac{k_{w, e x p} \cdot \eta_{w}}{\gamma_{w}}$ & 1 & Power law \\
'ST exp' & $\frac{k_{w, e x p} \cdot \eta_{w}}{\gamma_{w}}$ & 1 & Power law for $\dot{\gamma} \geq 1.51 / \mathrm{s}$ and \\
& & & Newton (plateau) for $\dot{\gamma}<1.51 / \mathrm{s}$ \\
'CM BK' & $\frac{D^{2} \cdot n^{3}}{150 \cdot(1-n)^{2}}$ & $\frac{25}{12}$ & Power law \\
'CM exp' & $\frac{k_{w, e x p} \cdot \eta_{w}}{\gamma_{w}}$ & $\frac{25}{12}$ & Power law \\
'CM exp*', & $\frac{k_{w, e x p} \cdot \eta_{w}}{\gamma_{w}}$ & $\frac{25}{12}$ & Power law \\
\hline
\end{tabular}

${ }^{a} T$ also applied to $L$ in Eq. 2 as $L^{*}=T \cdot L[\mathrm{~m}]$ zones, i.e. zones of lower concentration and thus reduced viscosity near the grain surface, especially pronounced with flexible macromolecules such as acrylamide-acrylate copolymers. Taking into account uncertainties about the specific value of the shift factor, especially with focus on implementation within the $\bar{v}-i$ plots (instead of $\dot{\gamma}$ vs. $\eta$ ), a correction parameter $\alpha^{*}$ is proposed in Eq. 2 resulting in $\frac{\Delta P}{2 \cdot L \cdot \kappa} \rightarrow \frac{\Delta P}{2 \cdot L \cdot \kappa \cdot \alpha^{*}}$ (cf. Eq. 5). This factor may be equally interpreted as a viscosity modifier $\left(\alpha^{*} \cdot \kappa\right)$ indicating a possible depletion by a vertical shift within the $\dot{\gamma}-\eta$ plot or as a shift between applied and 'in-pore' gradient (and therefore shear rate) $\left(\frac{1}{\alpha^{*}} \cdot \frac{\Delta P}{L}\right)$.

$$
\bar{v}_{\text {capillary }, O d W \alpha^{*}}=\left(\frac{m}{3 \cdot m+1}\right) \cdot R^{\frac{m+1}{m}} \cdot\left(\frac{\Delta P}{2 \cdot L \cdot \kappa \cdot \alpha^{*}}\right)^{\frac{1}{m}}
$$

\section{Materials and methods}

\subsection{Material characteristics}

Glass beads and sand were used as the granular host media in order to determine the influence of grain characteristics on the flow behaviour of polymer solutions through porous media. The glass beads are uniform with a diameter of $0.4-0.6 \mathrm{~mm}$. The material size was chosen to ensure a sufficient flow velocity at the considered maximum overpressure. The sand used was sieved to a grain size of 0.4-0.6 mm to obtain a comparable grain size distribution. The grain morphology of both glass beads and sand particles was studied exemplarily for a small selection of representative grains using a digital microscope (Fig. 2). The code introduced in [35] was used to analyse the shape and surface properties after applying a binary filter to obtain black and white images and a median filter to remove irregular artefacts. The results of the image processing and the characteristic parameters of glass beads and sand are summarised in Table 3.

For the preparation of the solutions, two synthetic anionic acrylamide-acrylate copolymer products (commercial name PHPA) equal in molecule type and degree of anionicity (30\%) were chosen, which only differ in molar mass (chain length): short = SM and long = LM. In addition, a variation of the concentration in solution was studied. Molar mass and concentration are given in Table 4. In order to minimize the influence of inherent variations of the polymer granules (dissolution velocity), the starting material was fractionated in dry sieves. The granules were then mixed according to a fixed particle size distribution into the solute. To prevent concentration fluctuations and polymer lumps, deionized water was first 

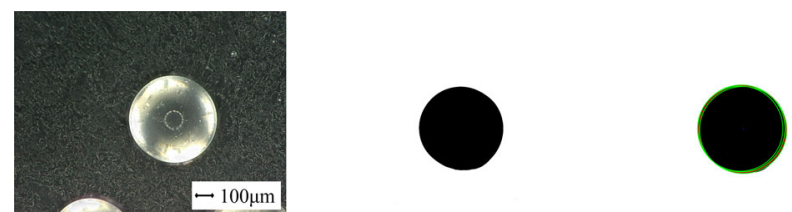

(a) Glass beads
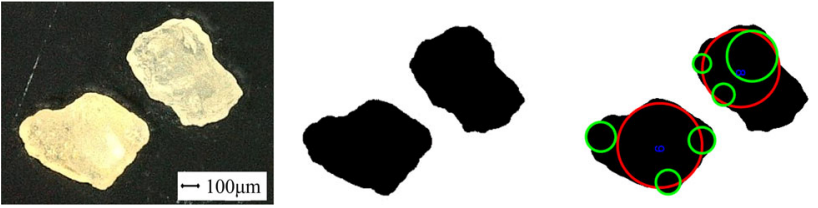

(b) Sand

Fig. 2 Image analyses, from left to right: raw image, post-processed image and MATLAB output according to [35]

stirred on a magnetic stirrer while the polymer was powdered onto the surface until all granules were hydrolysed and the solution became distinctly more viscous. The solution was then stirred at $500-550 \mathrm{rpm}$ with a blade paddle for 50 minutes and was then left to stand for $5 \mathrm{~min}$ $(\mathrm{SM})$ or $60 \mathrm{~min}(\mathrm{LM})$.

\subsection{Experimental setup and testing procedure}

The test setup used to study the permeation behaviour of polymer solutions is shown in Fig. 3 (not to scale). It represents an adaption for shear-thinning fluids from soil penetration testing standardized in $[16,17]$ and was specially designed for high viscosity fluids. In particular, the permeameter design encompasses a 1" connecting pipe, 0.5 " connections and a perforated plate (hole diameter 5 $\mathrm{mm}$ ) placed on a spacer ring to facilitate uniform inflow conditions to the soil sample with circular cross section. Potential head losses within the system can be considered insignificant. This was verified by means of a testing device with the same connecting equipment, but a larger soil cylinder equipped with pore pressure transducers, under similar boundary conditions with respect to soil, fluid and gradient. Hence, the applied gradients were calculated based on the total head difference (fluid head plus potential air overpressure) dissipating entirely along the
Table 4 Properties of polymers in solution

\begin{tabular}{lll}
\hline & Molar mass $[\mathrm{g} / \mathrm{mol}]$ & Concentration $[\mathrm{g} / \mathrm{l}]$ \\
\hline SM 0.75 & $5-7.5 \times 10^{6}$ & 0.75 \\
SM 1.50 & $5-7.5 \times 10^{6}$ & 1.50 \\
LM 0.75 & $22-27 \times 10^{6}$ & 0.75 \\
LM 1.50 & $22-27 \times 10^{6}$ & 1.50 \\
\hline
\end{tabular}

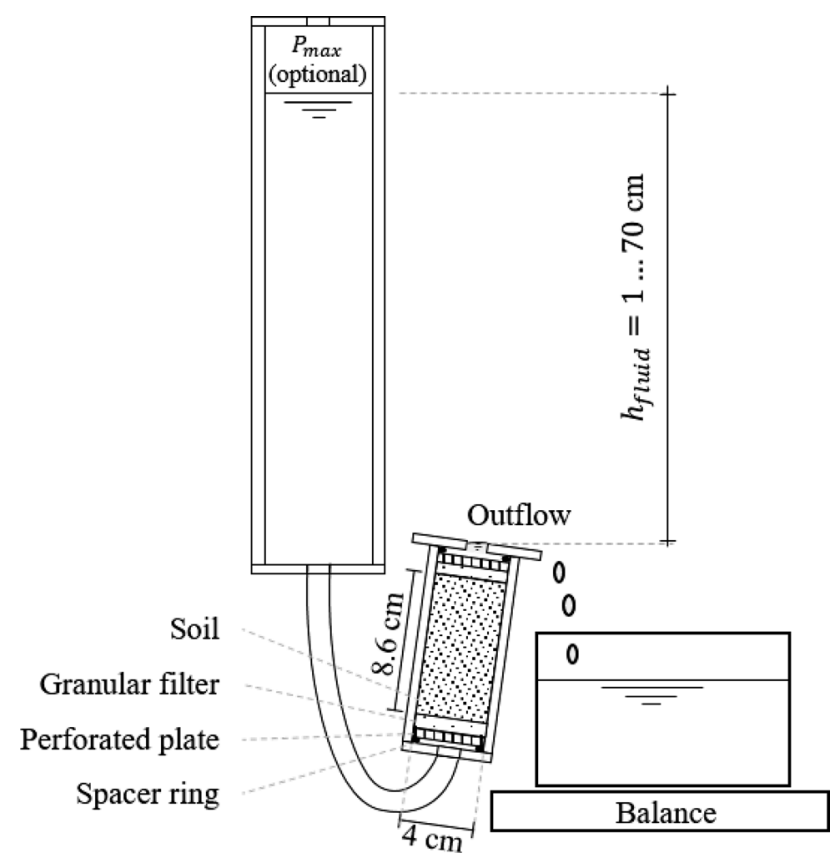

Fig. 3 Schematic of the test setup used

sample height with negligible influence from system boundary effects.

All tests were performed in a temperature-controlled room at $20{ }^{\circ} \mathrm{C}$. The amount of outflowing permeate was measured using a digital balance connected to a data logging system. Possible evaporation at inflow and outflow container fluid level was minimised by addition of a thin floating oil film.

A succession of stepwise constant gradients $i$ was applied in the order $10 / 7 / 4.5 / 1.9 / 1 / 0.5$, with the lowest gradient applied as a falling head. The stepwise decrease in

Table 3 Properties of the solids

\begin{tabular}{lllllll}
\hline & $\begin{array}{l}D \\
{[\mathrm{~mm}]}\end{array}$ & $\begin{array}{l}\rho_{s} / \rho_{d, \min } / \rho_{d, \max } \\
{\left[\mathrm{g} / \mathrm{cm}^{3}\right]}\end{array}$ & $\begin{array}{l}O \\
{[-]}\end{array}$ & $\begin{array}{l}\text { Sphericity } \\
\min / \max / \varnothing[-]\end{array}$ & $\begin{array}{l}k_{w} \\
{[\mathrm{~m} / \mathrm{s}]}\end{array}$ \\
\hline Glass & $0.4-0.6$ & $2.50 / 1.48 / 1.60$ & 0.99 & $0.97 / 1.00 / 0.98$ & $1.2 \times 10^{-4}$ \\
Sand & $0.4-0.6$ & $2.65 / 1.38 / 1.69$ & 0.37 & $0.71 / 0.94 / 0.80$ & $1.5 \times 10^{-4}$ & 0.41 \\
\hline
\end{tabular}

With $D$ grain size, $\rho_{s}$ specific density, $\rho_{d}$ dry density, $O$ roundness, $k_{w}$ hydraulic conductivity, $n$ porosity 
gradient envisages at best reflecting the environmental conditions of a support fluid penetrating into the ground at constant support fluid level, but decreasing gradient with progressing penetration.

Rheology tests of the polymer solutions were performed by means of a double gap coaxial cylinder with stepwise decreasing shear rate to best reflect the in-situ test conditions (decreasing flow velocity with decreasing gradient). The bulk rheology of the polymer solutions was determined both before and (for some samples also) after passing the permeameter.

A previously tested soil permeation device with a more complex construction closer to a classical permeation test following [17], in particular with more pipe connections, smaller pipe cross sections and thicker filter material, showed significant head losses within the system along the whole tested range of gradients and for all tested solutions compared to the results obtained with this device. It can therefore be concluded that classical permeation testing devices are not advisable for application with these types of fluids.

\section{Results and discussion}

\subsection{Bulk rheology tests}

\subsubsection{Polymer solutions as prepared}

The results of the bulk viscosity experiments conducted using the rheometer are given in Fig. 4. The viscosity plot of all tested polymer solutions (Fig. 4b) reflects a clear shear-thinning behaviour, showing a significant decrease in viscosity with increasing shear rate. A change in concentration visibly leads to a vertical curve shift. At higher shear rates, the viscosity values become independent of chain length (SM vs. LM) and the curves arrange according to concentration ( 0.75 vs. 1.50$)$, equally visible in Fig. $4 \mathrm{~b}$ for shear rates above $0.11 / \mathrm{s}$. At low shear rates, however, unlike LM, the SM solutions show a clear development of a Newtonian plateau, indicating the influence of the chain length on the onset of the Newtonian plateau.

The impact of concentration and molar mass (chain length) on the bulk viscosity obtained from rheometer measurements can be rationalized using the Flory-Huggins virial expansion as given in Eq. 6 (similar to that known for osmotic pressure, [23]), which is usually obtained from capillary viscosimeter tests for $\dot{\gamma} \rightarrow 0$. The equation will here be applied to all tested shear rates $(0.001-10001 / \mathrm{s})$. Flory-Huggins define a specific viscosity $\eta_{s p}$ (Eq. 7) as a relative viscosity value relating the polymer solution's viscosity $\eta$ to the solvent's viscosity $\eta_{0}$ (water). $[\eta]$ represents the intrinsic viscosity, i.e. the viscosity at a theoretical concentration $0 . k_{2}$ is an interaction coefficient. For simplicity and as the following coefficients $k_{3}, k_{4}$, etc. are negligibly small, the virial expansion is terminated after $k_{2}$ indicating a nearly quadratic increase of viscosity with concentration $c$. The interaction coefficient $k_{2}$ as well as the intrinsic visocisty $[\eta]$ can then be determined through linear regression within the $\eta_{s p} / c$ versus $c$ plot (Fig. 5a) for a given shear rate, where the gradient $\frac{\Delta\left(\eta_{s p} / c\right)}{\Delta c}$ yields the value of $k_{2}$ and the intercept of the regression line provides $[\eta]$ at $c \rightarrow 0$. The shear rate is displayed as brightness level. The $\dot{\gamma}_{\text {min }}=0.0011 / \mathrm{s}$ curves for both SM and LM are visible as the brightest lines at the bottom at $\frac{\eta_{s p}}{c}<10$.

$\frac{\eta_{s p}}{c}=[\eta]+k_{2} c+k_{3} c^{2} \cdots$

$\eta_{s p}=\frac{\eta-\eta_{0}}{\eta_{0}}$

The resulting parameters $[\eta]$ and $k_{2}$ are plotted as a function of shear rate $\dot{\gamma}$ in Fig. 5b for both polymers. Distinct differences between LM and SM can be observed for shear rates $\leq 0.11 / \mathrm{s}$. However, for shear rates above $0.11 / \mathrm{s}$, the differences between LM and SM visibly disappear, thus, the viscosity becomes a function solely of concentration $c$.

This observation may be explained by the coil model, where the polymers' viscosity mobilization occurs due to entanglement. The shear resistance is thereby created by the size of the cross section of the polymer molecules exposed to shear [14, 27]. At high shear rates, the polymer chains are arranged in a fully uncoiled parallel form providing minimal shear resistance against flow. Thus, viscosity is governed by concentration regardless of chain length (cf. Figs. 4 and 5 ). The effect of concentration is maintained with decreasing shear rates for each chain length. However, as polymer entanglement increases with decreasing shear stresses, shorter chains (SM) reach full coil entanglement at higher shear rates than longer chains (LM). This maximum entanglement coincides with a maximum viscosity (Newtonian plateau).

\subsubsection{Degradation considerations}

Figure 6 reflects the change of the measured bulk viscosity as induced by mechanical disturbance of the tested polymer solutions by soil permeation in the small flow channels of the glass beads (Fig. 6a) as well as age of the polymer granulate $(\approx 9$ months, Fig. $6 b)$. The effect was obtained by determination of the deviation of the rheometer viscosities as given by $f=\frac{\eta_{\text {end }}-\eta_{\text {start }}}{\eta_{\text {start }}}$, with $\eta_{\text {start }}$ representing the viscosity values of the fresh solution and $\eta_{\text {end }}$ the values after permeation or ageing, respectively. 


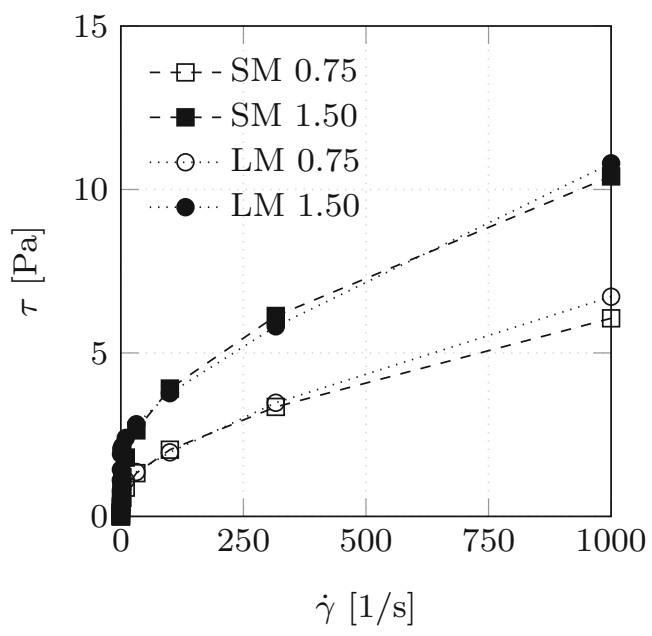

(a) Shear stress

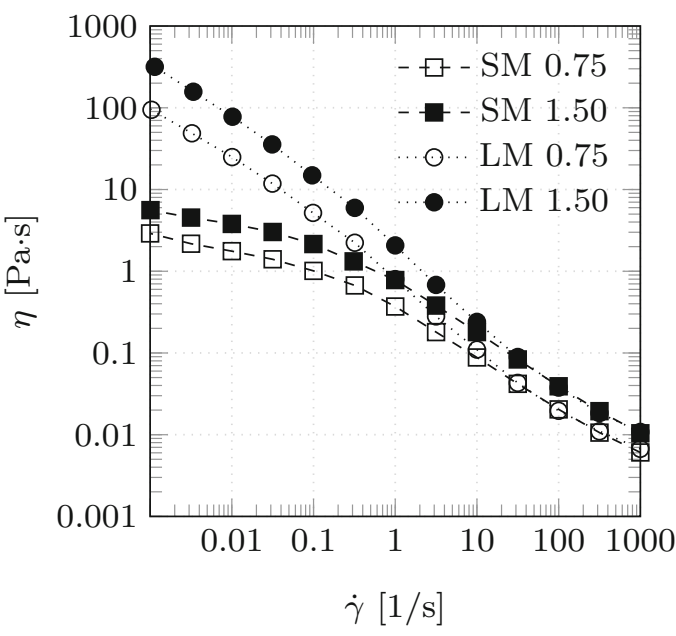

(b) Viscosity $(\eta=\tau / \dot{\gamma})$

Fig. 4 Experimental results of flow curves (rotational rheometer) of solutions with unaltered polymers

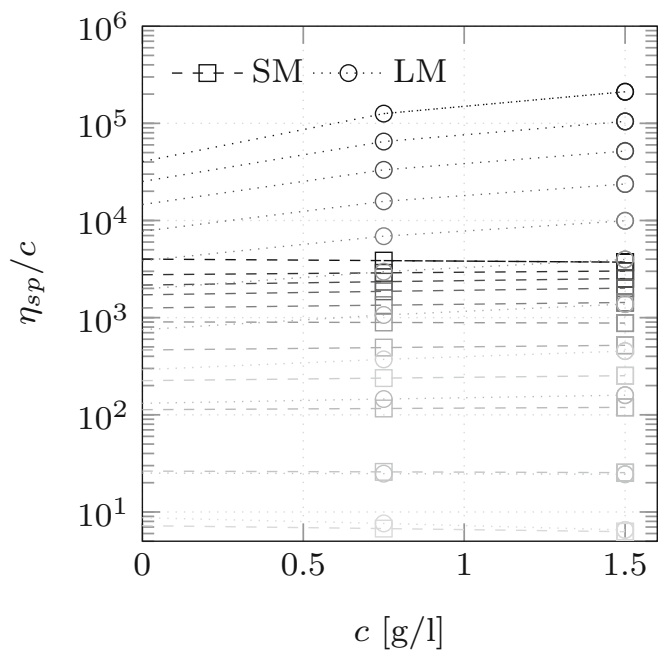

(a) Curves
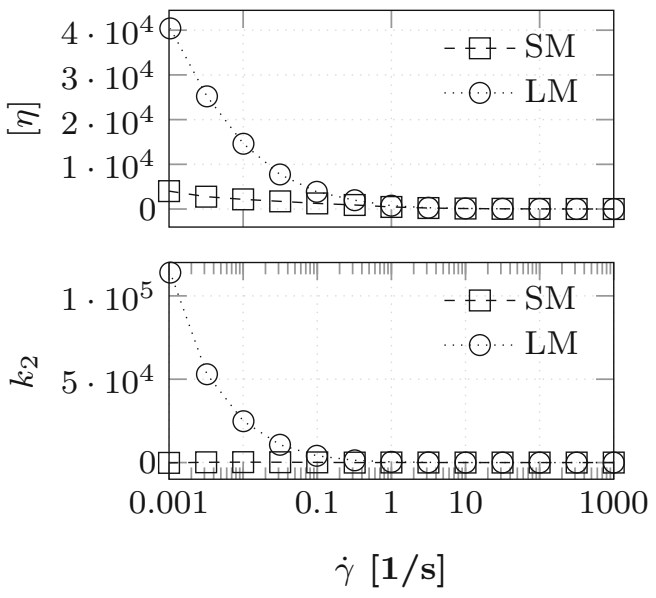

(b) Coefficients

Fig. 5 Flory-Huggins virial expansion from rheometer data according to Eqs. 6 and 7

A significant alteration $(|f|<66 \%)$ of the bulk viscosity was noted after the permeation of the glass beads despite their negligible surface roughness. With relation to the power-law parameters, this alteration amounts to a reduction of $\kappa$ to $0.65-0.7 \cdot \kappa$ and an increase of $m$ to $1.1-1.3 \cdot m$. It can be assumed that the additional effect of grain roughness (sand) induces a more pronounced occurrence of this effect.

The impact of a storage time of around 9 months had a similar influence on the polymer solutions with shorter chains. However, the impact was nearly doubled for the long-chain polymer solutions of the same concentration at all shear rates with $|f|<84 \%$. The linear increase of the viscosity alteration $|f|$ with decreasing shear rate for all fluids in the double-logarithmic plot (Fig. 4b) indicates mainly a degradation of the chains, but no significant reduction in polymer concentration, which would be visible at high shear rates. Two main causes for chain degradation in these cases are assumed to be mechanical, as induced by shearing energy (Fig. 6a) and chemical, as induced e.g., by oxidation during storage time (Fig. 6b) [28, 34].

\subsection{Flow tests}

The following Figs. 7 and 8 display the results of the experimental flow tests performed according to Sect. 3.2. 


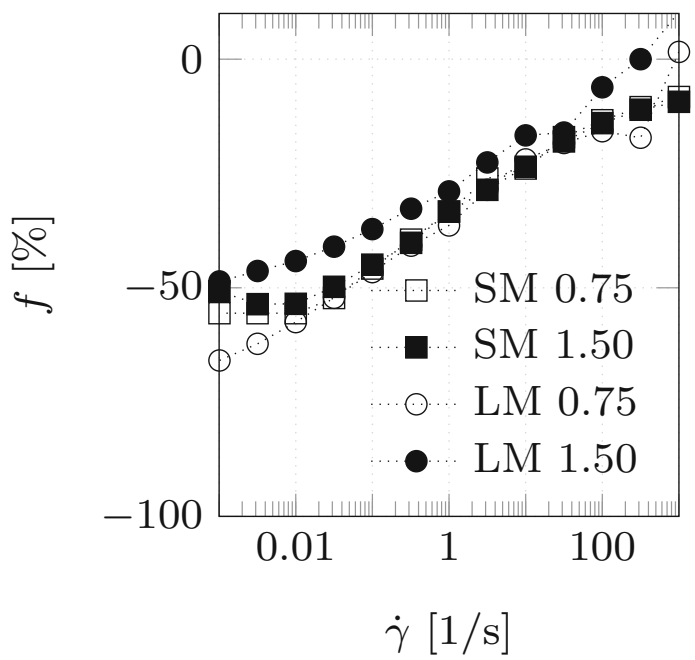

(a) Glass beads

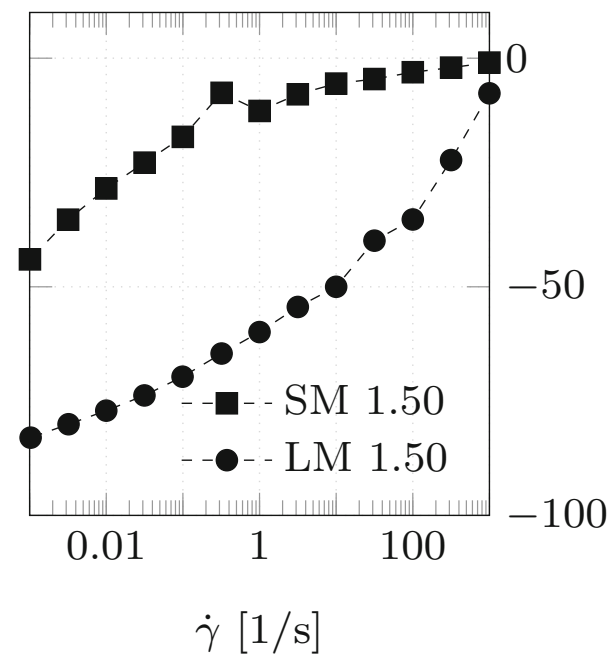

(b) Age

Fig. 6 Change of bulk rheology by $f=\frac{\eta_{\text {end }}-\eta_{\text {start }}}{\eta_{\text {start }}}[\%]$
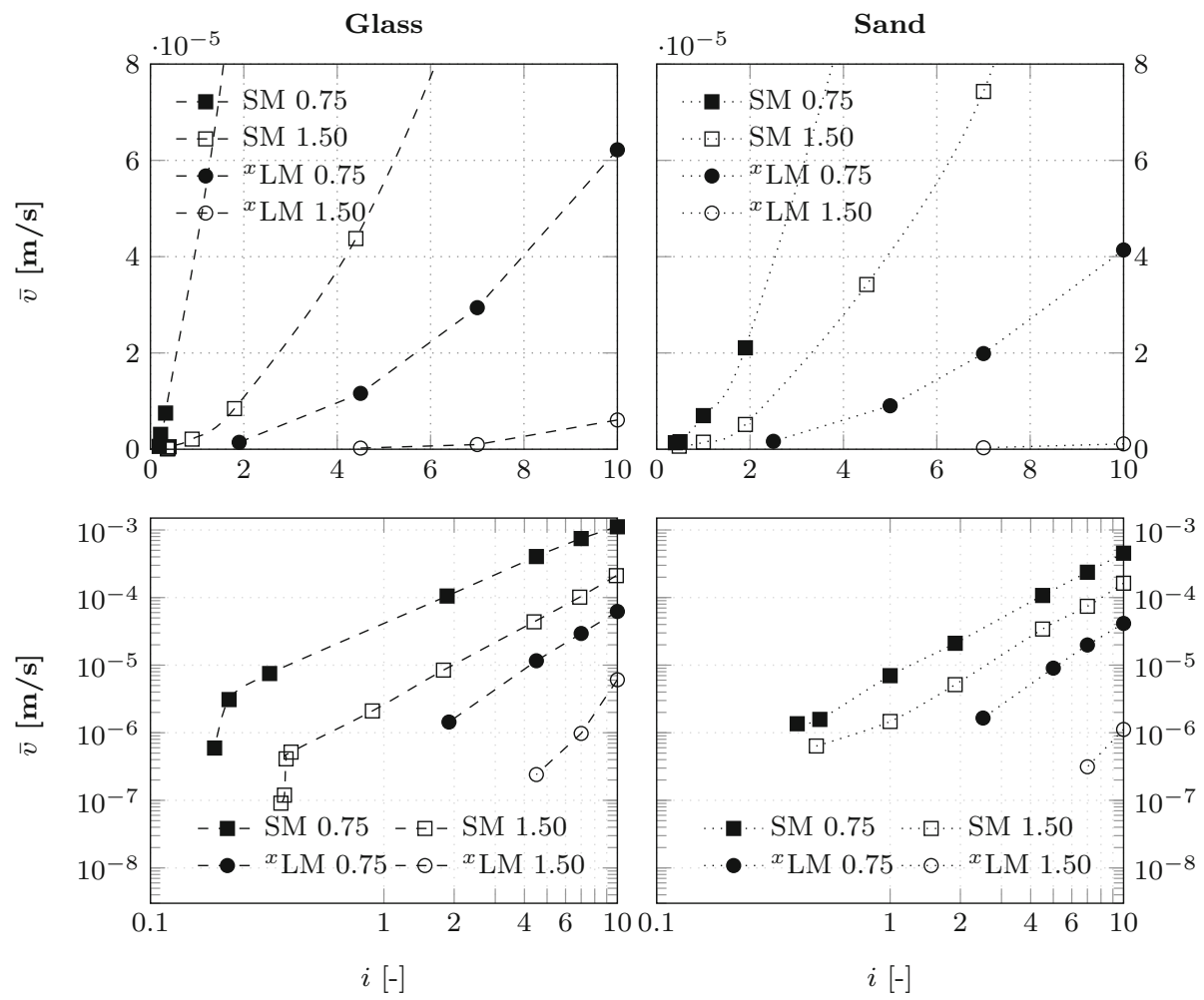

Fig. 7 Experimental results: filter velocity $\bar{v}$ with decreasing gradient $i$ (top: linear plot, bottom: $\log -\log$ plot)

Figure 7 shows the experimental results in terms of filter velocity at decreasing gradients in a linear as well as a double-logarithmic (log-log) scale for glass beads and sand, respectively. Figure 8 shows the experimental results in terms of capillary velocity $\bar{v}_{\text {capillary }}=\bar{v} / n$ separately for each polymer type.
For all LM curves, no outflow was observed below the lowest plotted gradient over a period of $40 \mathrm{~h}$. These curves are marked by ' $x$ ' indicating stagnation between the lowest plotted and the next applicable gradient. The other tests (SM) were stopped manually. While evaporation was minimised, especially values around and below approx. $\bar{v}=5 \times 10^{-7} \mathrm{~m} / \mathrm{s}$ with $\mathrm{LM}$ polymers are subject to 


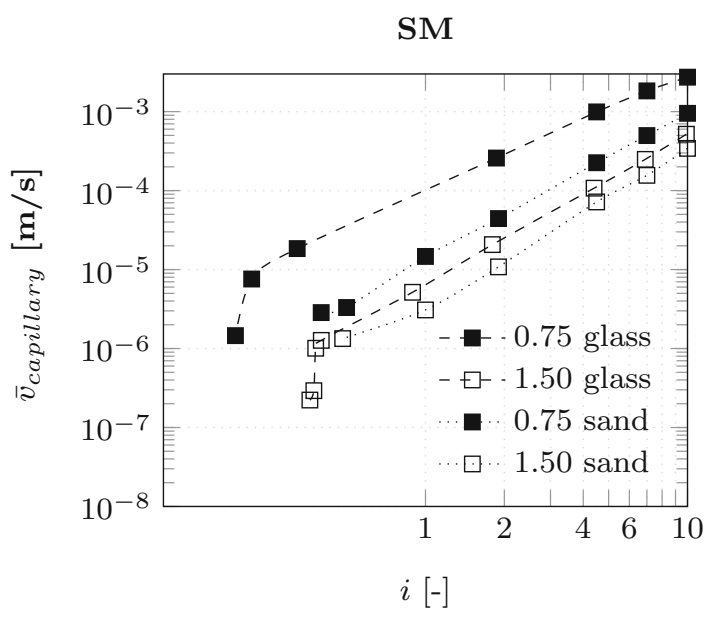

LM

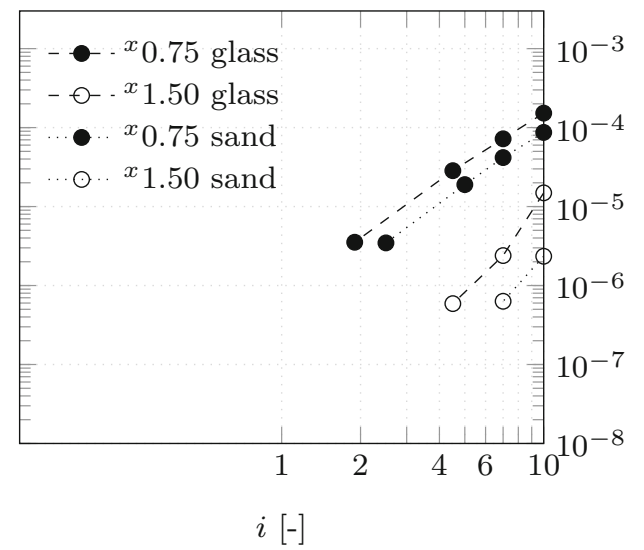

Fig. 8 Experimental results: interstitial velocity $\bar{v}_{\text {capillary }}=v / n$ with decreasing gradient $i$

measuring uncertainties. At these low flow rates, owing to the long chains with entanglement effects, the outflow occurs in large drops of several grammes only resulting in imprecise measurements and increased possibility of evaporation.

The visualisation for both porous materials in the linear plot shows an 'over-linear' relationship between the flow velocity and the hydraulic gradient for all curves, resulting in a decrease of the hydraulic conductivity $k=\bar{v} / i$ with decreasing gradient as opposed to Newtonian fluids such as water, where $\bar{v}$ is proportional to $i$ resulting in a constant value for $k$. This result reflects the shear-thinning behaviour of polymer solutions, that is, an increase in viscosity with decreasing shear rates and thus greater resistance to flow. At the higher range of tested gradients, the velocity curves are sorted in accordance with the rheometer curves at shear rates around 0.1 [1/s] (Fig. 1b). Hence, it may be concluded that the in-situ flow regime in this studied range of gradients does not reach a chain-length-independent viscosity controlled only by concentration.

In the double-log plot (Fig. 7 bottom), all curves essentially follow a nearly straight line indicating powerfunction behaviour. In addition to the observations with LM, the SM glass curves, too, exhibit an indication for stagnation, though at significantly lower gradient, with a significant drop in velocity within the last load step (falling gradient).

The capillary velocity plots shown in Fig. 8 highlight the impact of the porous medium. With all solutions tested, higher flow velocities can be observed in glass beads (dashed lines) as compared to sand (dotted lines). A comparison of the curve shapes shows relatively parallel curve shifts from glass beads to sand, i.e. a decrease in penetration velocity with an increase in surface roughness despite similar hydraulic conductivity with water $k_{w}$ (Table 3 ).
These observations can be explained by the influence of the interaction between grain morphology and polymer chains on the flow behaviour of the tested solutions in porous media. Sufficiently high concentrations as well as high molecule chain lengths result in molecule interaction and reduced polymer deformability (Sect. 4.1.1) and can thus contribute to a certain extent to flow and filtration mechanisms in porous media [11, 28, 36]. These effects can become more important in the narrow and winding pore channels of the rough porous medium than in rheometer flow tests with higher gap widths and smooth surface.

Following the theory of depleted zones [12, 27, 28], it is possible that there are slip zones of lower viscosity along the pore surface. However, the present results suggest that the soil roughness in the examined cases counteracts this effect. Consequently, at lower gradients, when the polymer chains get more coiled, stagnation is more likely to occur in the narrow flow paths with irregular rough surfaces. These effects cannot be captured by power-function approaches derived with parameters from rheometer tests, which is in accordance with the experimental results.

Generally, it can be concluded that both polymer and soil surface characteristics influence the penetration behaviour. In particular, an increase in polymer chain length and concentration decreases the penetration velocity at high gradients following the curvature of a power function in a $v-i$ plot. Pore surface roughness additionally markedly decreases the penetration velocity in the tested environments.

\subsection{Semi-empirical approaches for flow in porous media}

Figure 9 shows a comparison of the experimental results of Darcy velocity $\bar{v}$ against a decreasing gradient $i$ with their 
respective approximations for each polymer type (SM or LM) according to a general basic capillary bundle model as well as specific variations as summarised in Table 2. As a first step, no shift factor is considered, as the appropriate value is still unclear.

The respective power-law parameters $(m, \kappa)$ were determined as best-fit to the power-law region of the respective experimentally obtained rheometer curves for each test. However, the definition of one clear power-law region is not obvious with respect to the slightly curved course of viscosity as shown in Fig. $4 \mathrm{~b}$. Yet, it could be found that the slope of the $\bar{v}-i$ curves can be approximated relatively well by choosing a viscosity slope (power-law parameter $m$ ) matching the rheometer results at high shear rates (around 100-500 1/s). The approximation curves (dashdotted), apart from the ST model, then (with $m \approx 0.43-0.5$ ) match the inclination of the straight lines in the $\bar{v}-i \log -\log$ plot relatively well, i.e. run approximately in parallel to the experimental results, especially for higher hydraulic gradients.

But, as expected without considering a shift factor within the model, a significant deviation of the experimental velocity profile of $\Delta \bar{v} \approx 1 \times 10^{-1}-1 \times 10^{2} \mathrm{~m} / \mathrm{s}$ can be noted, which is significantly more pronounced with the SM solutions. Any increased velocity drop or stagnation at low hydraulic gradients cannot be captured by any of the applied models. The ST model, on the contrary, leads to an even flatter curve at a distinct point of deviation. Generally, it can be concluded that the deviations between approximation and experiment do not show a clear tendency for a best-fit model.

In order to differentiate the effects of the selected model variations (Table 2), a parametric study on their respective input parameters (Eq. 2) with respect to the $\bar{v}-i \log -\log$ plots is given in Fig. 10 based on the 'basic' capillary bundle model for SM 0.75 sand.

As given by their mathematical connection, a variation in $K, n, \kappa$ or $T$ results in a curve shift in the $\log -\log$ plot, while the power law exponent $m$ changes the inclination of the straight line in the $\log -\log$ plot. The critical shear rate $\dot{\gamma}_{\text {crit }}$ defines the gradient, at which Newtonian flow behaviour is assumed, resulting in a sudden change of slope of the $\bar{v}-i$ curve due to a sudden increase of $m$ to $m=1$. This critical gradient decreases with decreasing critical shear rate.

Overall, effects from soil parameter deviations $(K, n, T)$ are most pronounced for $K$. In particular, a prediction according to Blake-Kozeny $\left(K_{B K}\right)$ or Kozeny-Carman $\left(K_{K C}\right)$ increases the predicted flow velocity by several powers of ten, which in most cases surpasses the deviations found between model and experiment (Fig. 9). Reasonable variations in $n$ or $T$ cannot capture these deviations either.
Concerning fluid model parameters $\left(\kappa, m, \dot{\gamma}_{c r i t}\right)$, it can be noted that, while bulk rheology measurements showed a certain Newtonian plateau (Fig. 4b), this was not visible in the flow tests performed with sand or glass beads. Possibly, the combination of the tested concentrations and molar masses in the environment of the small pores of the tested materials did not enable full entanglement (Newtonian plateau formation) of the polymer chains at the tested velocities. A change of the power-law parameters from mechanical degradation as described in Sect. 4.1.2 and Fig. 6a would result in a markedly lower slope of all model curves due to an increase in $m$ and an upwards shift of every flow curve by $\Delta \bar{v}<1 \times 10^{1} \mathrm{~m} / \mathrm{s}$ due to a decrease of $\kappa$, both by approximately the same factor independent of the type of polymer solution. It is therefore unlikely that any explicable deviations in bulk rheological model parameters determined as best-fit parameters from rheometer tests could fully account for the deviations between approximation and experiment.

With the introduction of a shift factor $\alpha^{*}$ over $\bar{v}$, Fig. 11 shows the values for $\alpha^{*}$ which fit the experimental data best with reference to the capillary bundle basic model. With the exception of LM 1.50, overall, it can be seen that the $\alpha^{*}$ values to be considered are relatively constant along the measured velocities $\left(10^{-7}-10^{-3} \mathrm{~m} / \mathrm{s}\right)$. Moreover, naturally, the shift factors for glass bead environments are consistently lower compared to sand. The deviations in $\alpha^{*}$ for LM 1.50 along $\bar{v}$ (also LM 0.75 sand at lowest flow velocity) possibly result from measuring uncertainties at low flow rates (see above), i.e. the lower $\alpha^{*}$ are likely to be more accurate.

With respect to magnitudes, it can be observed that the SM tests show the lowest $\alpha^{*}$ values without marked impact by concentration. In contrast, a factor of approx. 2-3 can be deduced from $\alpha_{S M}^{*}$ to $\alpha_{L M 0.75}^{*}$ to $\alpha_{L M 1.50}^{*}$ showing a clear effect of chain length and concentration. It may be concluded that the SM chains, being sufficiently short, are still flexible enough in the porous environment to still form a pronounced slip effect (depletion). This effect is more and more reduced with chain length and especially with increased concentration obstructing free chain movement. That is, while pure bulk rheological power-law behaviour would underestimate penetration velocities significantly for SM and moderately for LM 0.75 , for LM 1.5 , it evidently overestimates the observed flow velocities resulting in a shift factor $\alpha^{*}>1$.

Overall, it can be concluded that the basic capillary bundle approach is capable of predicting the flow velocities of polymer solutions in the studied types of porous media over a range of $5 \cdot 10^{-7}-2 \cdot 10^{-3} \mathrm{~m} / \mathrm{s}=0.18 \mathrm{~cm} / \mathrm{h}-7.2 \mathrm{~m} /$ $\mathrm{h}$ with adequate accuracy if a specific constant shift factor is taken into account reflecting a systematic interaction 


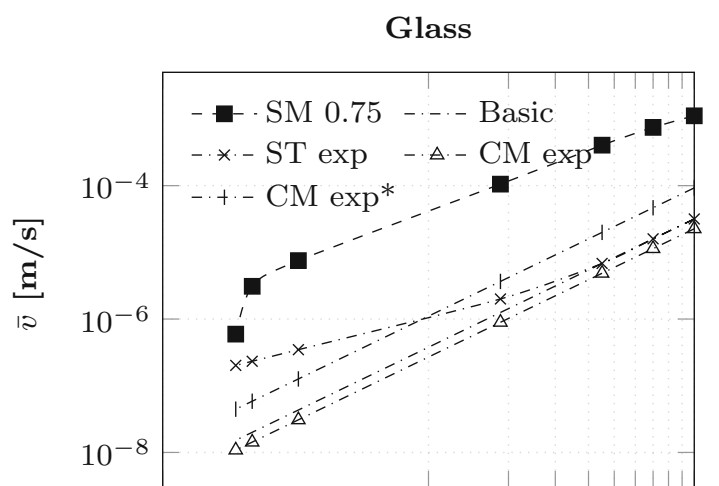

Sand
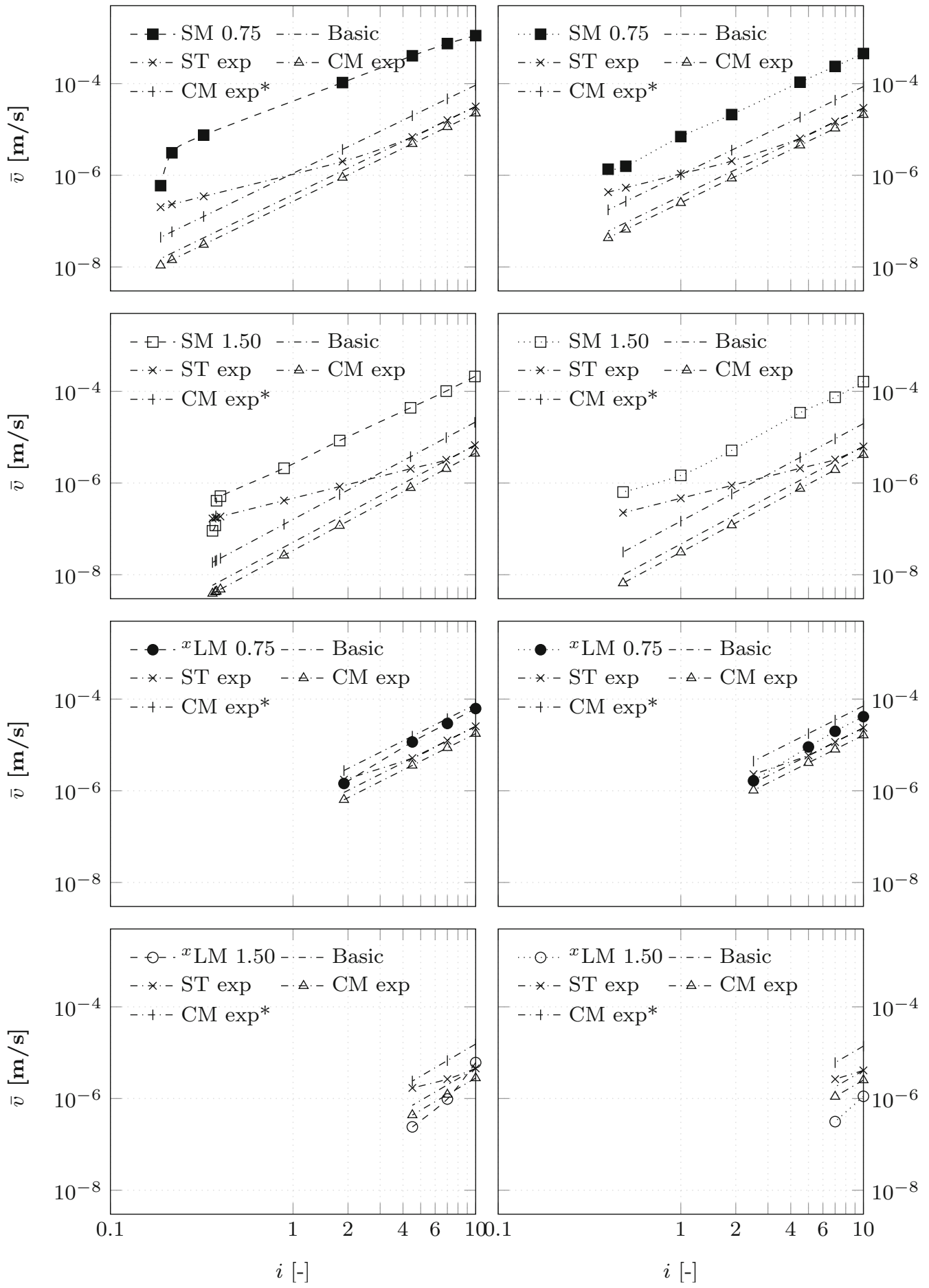

Fig. 9 Exemplary comparison of experiment and approximations $-\bar{v}$ versus $i$

between the pore morphology, the polymer chain length and the polymer concentration in solution. At flow velocities below $5 \times 10^{-7} \mathrm{~m} / \mathrm{s}=0.18 \mathrm{~cm} / \mathrm{h}$, stagnation can be assumed in most cases, which cannot be captured by this model adaption. 

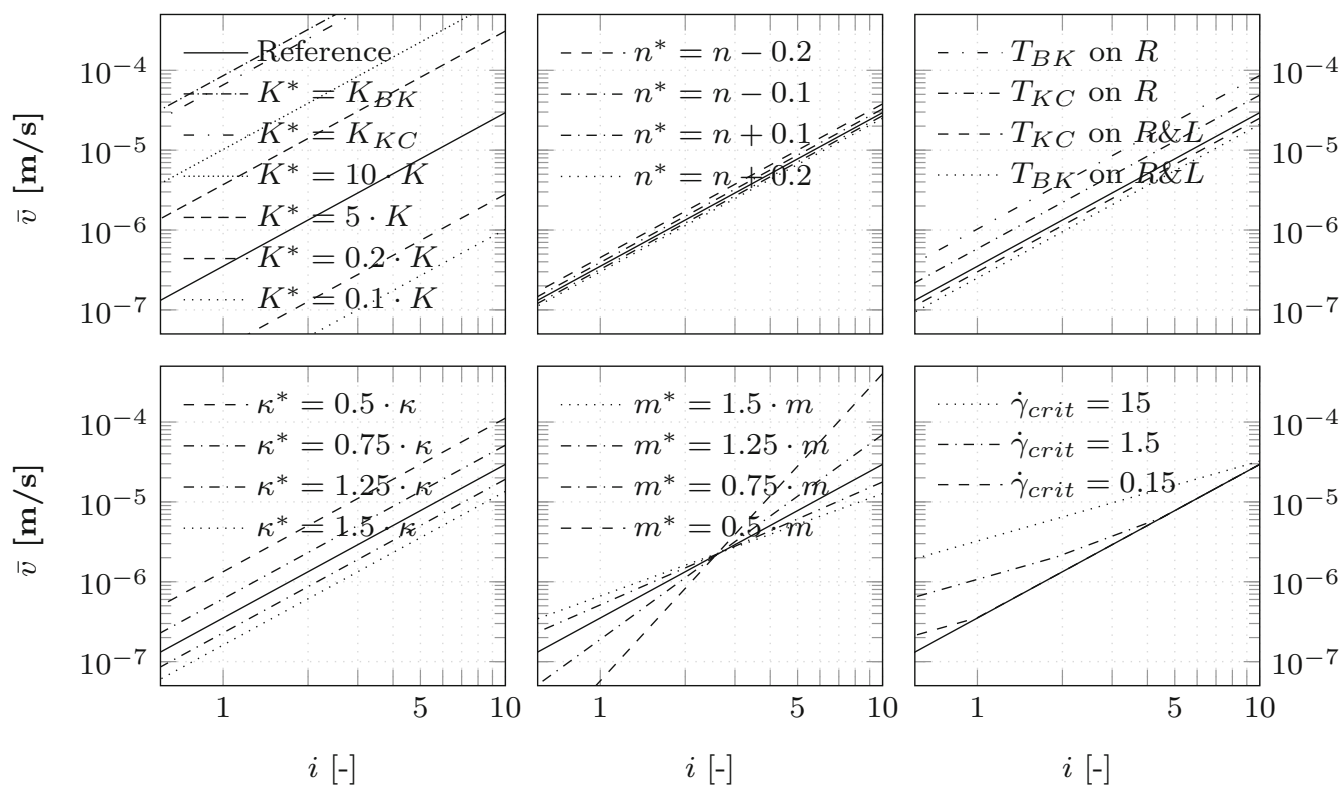

Fig. 10 Filter velocity $\bar{v}$ versus gradient $i$-parameter variation for the capillary bundle approximations based on the basic approach for SM 0.75 sand

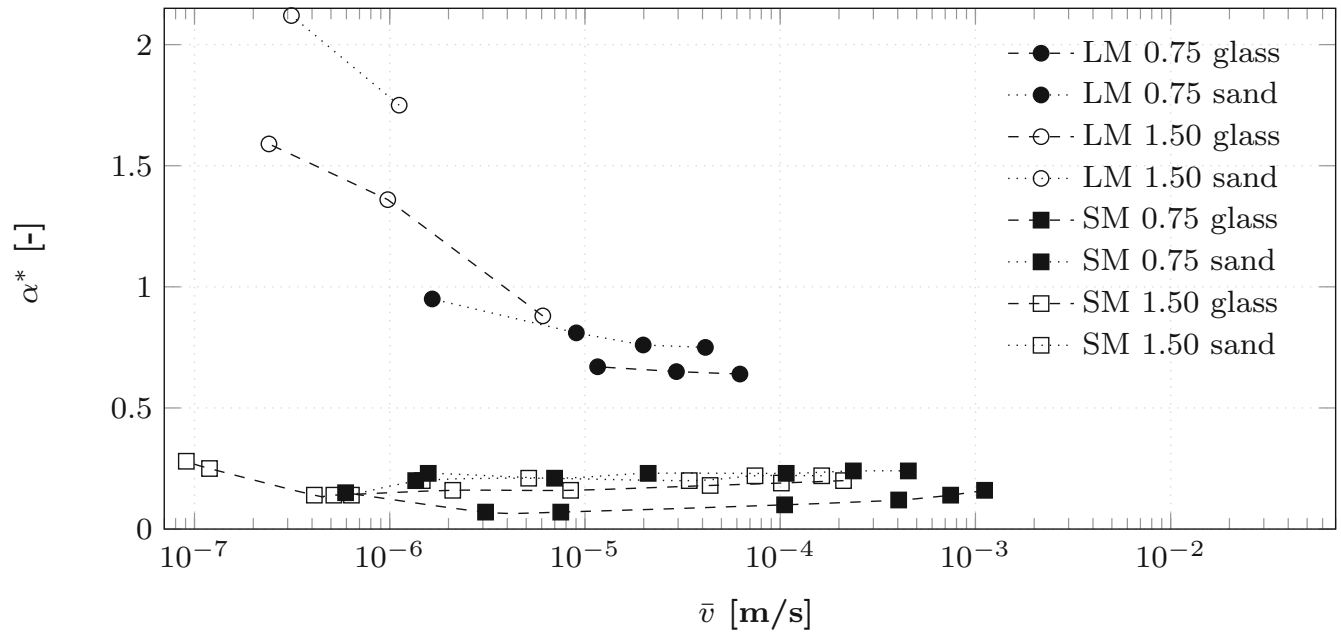

Fig. 11 Model factor $\alpha^{*}$ with reference to the basic model

\section{Practical implications}

Stability checks are an important tool for the prediction of possible collapse mechanisms of fluid-supported earth walls as well as economical precalculations for piles or shafts constructed with fluid support $[2,21,22,26,29,31,32]$. If filter velocities can indeed be approximated with sufficient accuracy by capillary bundle approaches with empirical interaction factors such as $\alpha^{*}$ down to around $=0.18 \mathrm{~cm} / \mathrm{h}$, then these models can be considered a suitable basis for existing formulations for stability checks, as the corresponding penetration rates for $\bar{v}<0.18 \mathrm{~cm} / \mathrm{h}$ are practically negligible for temporary fluid support.
Concerning the determination of the model parameters, $K\left(k_{w}\right), \kappa, m$ and $\alpha^{*}$ are the most important parameters, for which realistic variabilities need to be known. Permeability tests with water should form the basis of calculations for $K$. For polymers of similar origin and in the presented range of molar mass (which should comprise most viscosifying polymers used for fluid support) and for ground conditions with flow velocities similar or higher, simple viscosimeter measurements at higher shear rates $(\dot{\gamma}>1001 / \mathrm{s})$ should be sufficient for their approximation. The above results indicate that power-law behaviour is sufficient to predict the qualitative curvature of the flow behaviour in the tested gradient range as no Newtonian plateau was measurable. 


\section{Conclusion}

The increasing use of polymer solutions as support fluids demands a more detailed discussion of their penetration behaviour and prediction thereof with special focus on small flow velocities. The present paper has shown that simple viscosity-based capillary bundle models are able to depict rather adequately the qualitative slope of the $\bar{v}-i$ flow curve for filter velocities above $\bar{v}=5 \times 10^{-7} \mathrm{~m} / \mathrm{s}$.

Thereby, assumptions for tortuosity or permeability, e.g. based on Kozeny, or a more precise representation of bulk rheological flow behaviour, e.g. by [29], were not able to capture the complex interaction and lead to significant over- or underestimations of the experimentally determined flow velocities in some cases. In fact, the deviations between model and experiment could be explained by interactions between the flow path morphology and the polymer chains. These interactions could be accounted for by an empirical model constant $\alpha^{*}$, following approaches. e.g. summarised by [28] or [27]. In addition to these findings, $\alpha^{*}$ was found to depend on polymer chain length and concentration as well as soil morphology.

Taking under consideration this correction factor, capillary bundle approximations can be considered as sufficiently accurate for global stability calculations, even though they are unable to predict a complete stagnation of the fluid, which was observed at low gradients with higher polymer chain length, concentration and surface roughness of the granular material.

The experimental investigations presented here can be regarded as the first systematic steps of a broader analysis on the prediction of penetration rates based on the soil and polymer nature (e.g. pore diameters, grain pattern, polymer types). Subsequent studies with focus on economical factors could not only take into special consideration the multi-phase interaction between soil and polymer at low gradients, but also possible mineral surface or polymeric charges influencing possible pore-blocking effects.

Funding Open Access funding enabled and organized by Projekt DEAL.

\section{Declarations}

Conflict of interest The authors declare that they have no conflict of interest.

Open Access This article is licensed under a Creative Commons Attribution 4.0 International License, which permits use, sharing, adaptation, distribution and reproduction in any medium or format, as long as you give appropriate credit to the original author(s) and the source, provide a link to the Creative Commons licence, and indicate if changes were made. The images or other third party material in this article are included in the article's Creative Commons licence, unless indicated otherwise in a credit line to the material. If material is not included in the article's Creative Commons licence and your intended use is not permitted by statutory regulation or exceeds the permitted use, you will need to obtain permission directly from the copyright holder. To view a copy of this licence, visit http://creativecommons. org/licenses/by/4.0/.

\section{References}

1. Alexander-Katz A, Schneider MF, Schneider SW, Wixforth A, Netz RR (2006) Shear-flow-induced unfolding of polymeric globules. Phys Rev Lett 97(13):138101-1-138101-4

2. Alexanderson M (2001) Construction of a cable tunnel ID 2-6 Vibhavadi. In: Proceedings of 5th international symposium on microtunnelling. Bauma, Munich, pp 139-147

3. Bird RB, Stewart WE, Lightfoot EN (2007) Transp. Phen. Wiley, New York

4. Blake FC (1922) The resistance of packing to fluid flow. Trans Am Inst Chem Eng 14:415-421

5. Borghi FX (2006) Soil conditioning for pipe jacking and tunnelling. Ph.D. thesis, University of Cambridge. https://doi.org/10. 17863/CAM.19109

6. Brazel CS, Rosen SL (2012) Fundamental principles of polymeric materials. Wiley, Hoboken

7. Cannella W, Huh C, Seright R (1988) Prediction of xanthan rheology in porous media. In: SPE annual technical conference and exhibition. Society of Petroleum Engineers. https://doi.org/ 10.2118/18089-ms

8. Carman PC (1937) Fluid flow through granular beds. Trans Inst Chem Eng 15:150-166

9. Carreau RJ (1968) Rheological equations of state from molecular network theories. PhD thesis, University of Wisconsin, Madison

10. Carrier WD (2003) Goodbye, Hazen; Hello, Kozeny-Carman. J Geotech Geoenviron 129(11):1054-1056. https://doi.org/10. 1061/(ASCE)1090-0241(2003)129:11(1054)

11. Chauveteau G (1986) Fundamental criteria in polymer flow through porous media and their importance in the performance differences of mobility-control buffers. Water Solub Polym 213:227-268

12. Chauveteau G, Tirrell M, Omari A (1984) Concentration dependence of the effective viscosity of polymer solutions in small pores with repulsive or attractive walls. J Colloid Interface Sci 100(1):41-54. https://doi.org/10.1016/0021-9797(84)90410-7

13. Christopher RH, Middleman S (1965) Power-law flow through a packed tube. Ind Eng Chem Fundam 4(4):422-426. https://doi. org/10.1021/i160016a011

14. Colby RH, Boris DC, Krause WE, Dou S (2007) Shear thinning of unentangled flexible polymer liquids. Rheol Acta 46(5):569-575. https://doi.org/10.1007/s00397-006-0142-y

15. Darcy H (1856) Les fontaines publiques de la ville de Dijon: Exposition et application des principes à suivre et des formules à employer dans les questions de distribution d'eau. Victor Dalmont, Paris

16. DIN 4127:2014: Earthworks and foundation engineering - test methods for supporting fluids used in the construction of diaphragm walls and their constituent products; German version. Standard, Deutsches Institut für Normung, Beuth Verlag

17. EN ISO 17892-11:2019: Geotechnical investigation and testing laboratory testing of soil - part 11: permeability tests; German version EN ISO 17892-11:2019. Standard, Deutsches Institut für Normung, Beuth Verlag

18. Hirasaki GJ, Pope GA (1974) Analysis of factors influencing mobility and adsorption in the flow of polymer solution through 
porous media. Soc Petr Eng J 14(04):337-346. https://doi.org/10. 2118/4026-PA

19. Kawale D, Marques E, Zitha PLJ, Kreutzer MT, Rossen WR, Boukany PE (2017) Elastic instabilities during the flow of hydrolyzed polyacrylamide solution in porous media: effect of pore-shape and salt. Soft Matter 13(4):765-775. https://doi.org/ $10.1039 / \mathrm{c} 6 \mathrm{sm} 02199 \mathrm{a}$

20. Kozeny J (1927) Über kapillare Leitung des Wasser im Boden: Aufstieg, Versickerung und Anwendung auf die Bewässerung. Wien Akad Wiss 136:271-306

21. Lam C, Jefferis S (2018) Polymer support fluids in civil engineering. ICE Publishing, London

22. Lesemann H, Vogt N, Pulsfort M (2016) Analytical stability checks for diaphragm wall trenches and boreholes supported by polymer solutions. In: Proceedings of 13th Baltic sea geotechnical conference, Lithuania, pp 231-238

23. Lieske W, Baille W, Tripathy S, Schanz T (2020) An alternative approach for determining suction of polyethylene glycols for soil testing. Géot lett 10(1):1-5. https://doi.org/10.1680/jgele.19. 00017

24. Lohne A, Nødland O, Stavland A, Hiorth A (2017) A model for non-Newtonian flow in porous media at different flow regimes. Comput Geosci 21(5-6):1289-1312. https://doi.org/10.1007/ s10596-017-9692-6

25. Ostwald W (1929) Ueber die rechnerische Darstellung des Strukturgebietes der Viskosität. Kolloid-Zeitschr 47(2):176-187. https://doi.org/10.1007/BF01496959

26. Ouyang Y, Jefferis S, Wiltcher P (2018) A major infrastructure project and the formation of the UK's largest rotary pile under polymer support fluid. In: Proceedings of DFI-EFFC international conference on deep foundations and ground improvement, Rome, pp 541-550

27. Skauge A, Zamani N, Gausdal Jacobsen J, Shaker Shiran B, AlShakry B, Skauge T (2018) Polymer flow in porous media: relevance to enhanced oil recovery. Colloids Interfaces 2(3):27. https://doi.org/10.3390/colloids2030027

28. Sorbie KS (1991) Polymer-improved oil recovery. Blackie, Glasgow

29. Steinhoff J (1993) Standsicherheitsbetrachtungen für polymergestützte Erdwände. Ph.D. thesis, Bergische Universität Wuppertal, Wuppertal

30. Stewart DP, Chen YR, Kutter BL (1998) Experience with the use of methylcellulose as a viscous pore fluid in centrifuge models. Geot Test J 21(4):365-369

31. Verst R, Pulsfort M (2019) Stability analysis for tunnel faces supported by means of polymer solutions. Taylor \& Francis, pp 3299-3308. https://doi.org/10.1201/9780429424441-349

32. Wheeler P (2003) Clear as mud. New Civil Eng

33. Wreath D, Pope GA, Sepehrnoori K (1990) Dependence of polymer apparent viscosity on the permeable media and flow conditions. In Situ 14(3):263-284

34. Yu JFS, Zakin JL, Patterson GK (1979) Mechanical degradation of high molecular weight polymers in dilute solution. J Appl Polym Sci 23(8):2493-2512. https://doi.org/10.1002/app.1979. 070230826

35. Zheng J, Hryciw RD (2015) Traditional soil particle sphericity, roundness and surface roughness by computational geometry. Géotechnique 65(6):494-506. https://doi.org/10.1680/geot.14.P. 192

36. Zitha PL, Chauveteau G, Léger L (2001) Unsteady-state flow of flexible polymers in porous media. J Colloid Interface Sci 234(2):269-283

Publisher's Note Springer Nature remains neutral with regard to jurisdictional claims in published maps and institutional affiliations. 